\title{
5-Hydroxymethylcytosine signatures in circulating cell-free DNA as diagnostic biomarkers for human cancers
}

\begin{abstract}
Wenshuai $\mathrm{Li}^{1,{ }^{*}}$, Xu Zhang ${ }^{2, *}$, Xingyu Lu ${ }^{3,4, *}$, Lei You, ${ }^{5, *}$, Yanqun Song ${ }^{4}$, Zhongguang Luo ${ }^{1}$, Jun Zhang ${ }^{1}$, Ji Nie ${ }^{3}$, Wanwei Zheng ${ }^{1}$, Diannan $\mathrm{Xu}^{1}$, Yaping Wang ${ }^{6}$, Yuanqiang Dong ${ }^{6}$, Shulin $\mathrm{Yu}^{7}$, Jun Hong ${ }^{6}$, Jianping Shi ${ }^{8}$, Hankun Hao ${ }^{6}$, Fen Luo ${ }^{6}$, Luchun Hua ${ }^{6}$, Peng Wang ${ }^{7}$, Xiaoping Qian ${ }^{9}$, Fang Yuan ${ }^{10,1}$, Lianhuan Wei ${ }^{11}$, Ming Cui ${ }^{5}$, Taiping Zhang, ${ }^{5}$ Quan Liao ${ }^{5}$, Menghua Dai ${ }^{5}$, Ziwen Liu ${ }^{5}$, Ge Chen ${ }^{5}$, Katherine Meckel ${ }^{12}$, Sarbani Adhikari ${ }^{12}$, Guifang Jia ${ }^{11,13}$, Marc B Bissonnette ${ }^{12}$, Xinxiang Zhang ${ }^{10,11}$, Yupei Zhao ${ }^{5}$, Wei Zhang ${ }^{14}$, Chuan He ${ }^{3,10}$, Jie Liu ${ }^{1,15}$

${ }^{I}$ Department of Digestive Diseases, Huashan Hospital, Fudan University, Shanghai 200040, China, ${ }^{2}$ Section of Hematology/Oncology, Department of Medicine, University of Illinois, Chicago, IL 60612, USA; ${ }^{3}$ Department of Chemistry, Department of Biochemistry and Molecular Biology, and Institute for Biophysical Dynamics, Howard Hughes Medical Institute, The University of Chicago, Chicago, IL 60637, USA; ${ }^{4}$ Shanghai Epican Genetech, Co. Ltd., Zhangjiang Hi-Tech Park, Shanghai 201203, China; ${ }^{5}$ Department of General Surgery, Peking Union Medical College Hospital, Chinese Academy of Medical Sciences, Beijing 100730, China; ${ }^{6}$ Department of General Surgery, Huashan Hospital, Fudan University, Shanghai 200040, China; ${ }^{7}$ Department of Integrative Oncology, Shanghai Cancer Center, Fudan University, Shanghai 200032, China: ${ }^{8}$ Department of Digestive Diseases, Pudong Hospital, Fudan University, Shanghai 201399, China; ${ }^{9}$ Department of Oncology, Nanjing Drum Tower Hospital, Nanjing University Medical School, Nanjing 210008, China; ${ }^{10}$ Beijing National Laboratory for Molecular Sciences, College of Chemistry, Peking University, Beijing 100871, China; ${ }^{11}$ Key Laboratory of Bioorganic Chemistry and Molecular Engineering of Ministry of Education, Peking University, Beijing 100871, China; ${ }^{12}$ Department of Medicine, The University of Chicago, Chicago, IL 60637, USA; ${ }^{13}$ Department of Chemical Biology, Structure and Function Biomolecules Center, College of Chemistry and Molecular Engineering, Peking University, Beijing 100871, China; ${ }^{14}$ Department of Preventive Medicine and The Robert H. Lurie Comprehensive Cancer Center, Northwestern University Feinberg School of Medicine, Chicago, IL 60611, USA; ${ }^{15}$ Department of Immunology, State Key Laboratory of Genetic Engineering, Institutes of Biomedical Sciences, Fudan University, Shanghai 200433, China
\end{abstract}

DNA modifications such as 5-methylcytosine $(5 \mathrm{mC})$ and 5-hydroxymethylcytosine $(5 \mathrm{hmC})$ are epigenetic marks known to affect global gene expression in mammals. Given their prevalence in the human genome, close correlation with gene expression and high chemical stability, these DNA epigenetic marks could serve as ideal biomarkers for cancer diagnosis. Taking advantage of a highly sensitive and selective chemical labeling technology, we report here the genome-wide profiling of $5 \mathrm{hmC}$ in circulating cell-free DNA (cfDNA) and in genomic DNA (gDNA) of paired tumor and adjacent tissues collected from a cohort of 260 patients recently diagnosed with colorectal, gastric, pancreatic, liver or thyroid cancer and normal tissues from 90 healthy individuals. $5 \mathrm{hmC}$ was mainly distributed in transcriptionally active regions coincident with open chromatin and permissive histone modifications. Robust cancer-associated $5 \mathrm{hmC}$ signatures were identified in cfDNA that were characteristic for specific cancer types. $5 \mathrm{hmC}$-based biomarkers of circulating cfDNA were highly predictive of colorectal and gastric cancers and were superior to conventional biomarkers and comparable to $5 \mathrm{hmC}$ biomarkers from tissue biopsies. Thus, this new strategy could lead to the development of effective, minimally invasive methods for diagnosis and prognosis of cancer from the analyses of blood samples.

Keywords: 5-hydroxymethylcytosine; liquid biopsy; circulating cell-free DNA; cancer biomarker; 5hmC-Seal Cell Research (2017) 27:1243-1257. doi:10.1038/cr.2017.121; published online 19 September 2017

*These four authors contributed equally to this work.

Correspondence: Chuan $\mathrm{He}^{\mathrm{a}}$, Jie Liu ${ }^{\mathrm{b}}$, Wei Zhang ${ }^{\mathrm{c}}$

aE-mail: chuanhe@uchicago.edu

bE-mail: jieliu@fudan.edu.cn

${ }^{c}$ E-mail: wei.zhang1@northwestern.edu

Received 24 July 2017; revised 6 August 2017; accepted 9 August 2017; published online 19 September 2017

\section{Introduction}

Cytosine methylation (formation of 5-methylcytosine, $5 \mathrm{mC}$ ) is a well-established epigenetic mechanism that affects global gene expression $[1,2]$. The $5 \mathrm{mC}$ remodeling of DNA is used extensively during mammalian devel- 
opment and cell differentiation, as well as during cancer initiation, progression and in the therapeutic response [3, 4]. Active demethylation in the mammalian genome is mediated by the TET (Ten-Eleven Translocation) family of dioxygenases that oxidize the $5 \mathrm{mC}$ modification to 5 -hydroxymethylcytosine $(5 \mathrm{hmC})[5,6]$, and further to 5 -formylcytosine (5fC), and 5-carboxylcytosine $(5 \mathrm{caC})$ [7-9]. The "intermediate" $5 \mathrm{hmC}$ not only marks active demethylation but also serves as a relatively stable DNA mark that plays distinct epigenetic roles [2, 10-15]. Recent genome-wide sequencing maps of $5 \mathrm{hmC}$ in various mammalian cells and tissues support its role as a marker for gene expression [16-22]; it is enriched in enhancers, gene-bodies and promoters, and changes in $5 \mathrm{hmC}$ correlate with changes in gene expression levels [22, 23].

The discovery of cell-free DNA (cfDNA) originating from different tissues in the circulating blood has revolutionary potential for the clinic [24]. Liquid biopsy-based biomarkers and detection tools offer substantial advantages over existing diagnostic and prognostic methods, including being minimally invasive. They thus have a cost-efficient potential to promote higher patient compliance and clinical convenience to enable dynamic monitoring [25]. Tumor-related somatic mutations in cfDNA have been shown to be shared with the tumor tissue, although low mutation frequency and the lack of information on tissue of origin hamper the detection sensitivity. $5 \mathrm{mC}$ and $5 \mathrm{hmC}$ in cfDNA from liquid biopsies could serve as parallel or more valuable biomarkers for non-invasive diagnosis and prognosis of human diseases, because they recapitulate gene expression changes in relevant cell states. If these cytosine modification patterns can be sensitively detected, disease-specific biomarkers could be identified for effective early detection, diagnosis and prognosis.

High-throughput sequencing is an ideal platform for detecting genome-wide cytosine modification patterns. Whole-genome bisulfite sequencing or alternative reduced representative methods have been applied in biomarker research with cfDNA [26-28]. Tissue- and cancer- specific methylation sites have shown promising performance in tracking tissue-of-origin from circulating blood $[26,28]$. However, $5 \mathrm{mC}$ serves mostly as a repressive mark with a high background level in the human genome, and its sequencing with bisulfite treatment has been hampered with extensive DNA degradation, in particular with cfDNA. Taking advantage of the presence of the hydroxymethyl group, selective chemical labeling can be applied to map $5 \mathrm{hmC}$ using low levels of DNA with high sensitivity. The profiling method is robust and cost-effective for studies of large cohorts and practical applications. Here, we have established $5 \mathrm{hmC}$-Seal technology for $5 \mathrm{hmC}$ profiling in cfDNA. We show that the differentially enriched $5 \mathrm{hmC}$ regions in cfDNA are excellent markers for solid tumors.

\section{Results}

\section{$5 \mathrm{hmC}$-Seal profiling in clinical specimens}

We first optimized our previously published nanohmC-Seal profiling method [29] (Figure 1) for cfDNA. The adaptor was pre-ligated with barcodes to enhance the efficiency of library construction and decrease cross contamination between large cohorts of samples. The labeling, binding and washing steps were optimized for capturing limited $5 \mathrm{hmC}$-containing cfDNA fragments. We profiled $5 \mathrm{hmC}$ in plasma cfDNA from cancer patients and healthy controls, as well as in genomic DNA (gDNA) isolated from tumors and adjacent healthy tissues. We sampled 90 healthy individuals, 260 cancer patients and 71 patients with benign diseases among Chinese populations (Supplementary information, Tables S1 and S2). For these patients and healthy controls, the study generated $4015 \mathrm{hmC}-$ Seal libraries from plasma cfDNA and 192 $5 \mathrm{hmC}$-Seal libraries from tissue gDNA (Supplementary information, Table S3). The cohort samples were collected and profiled in three batches (Supplementary information, Table S3). To minimize the influence of an experimental batch effects, differential $5 \mathrm{hmC}$ between cancers and controls (or between tumors and adjacent tissues) was analyzed with the first (discovery) batch and validated in the second (validation) and third (additional validation) batches.

To validate the $5 \mathrm{hmC}$ capture efficiency and the reliability of the modified assay, we spiked a pair of synthetic DNA probes containing and not-containing $5 \mathrm{hmC}$ into plasma cfDNA. The $5 \mathrm{hmC}$-Seal capture generated an average of 56-fold $5 \mathrm{hmC}$ enrichment of the spiked-in probes compared to control without pull-down (Supplementary information, Figure S1A). Samples with physiologically relevant amounts of cfDNA $(1,2,5,10,20 \mathrm{ng})$ and $5 \mathrm{hmC}$-containing spike-in probes at a fixed amount of $2.6 \mathrm{fg}$ were processed and sequenced, respectively. A linear relationship was observed between the proportion of $5 \mathrm{hmC}$-containing spike-in readouts and the spike-in concentration within cfDNA $\left(r^{2}=0.99\right.$, Supplementary information, Figure S1B), confirming the quantitative capture of $5 \mathrm{hmC}$ even down to $1 \mathrm{ng}$ of input cfDNA.

\section{Global and genomic distribution of $5 \mathrm{hmC}$ modifications}

We evaluated the global $5 \mathrm{hmC}$ level variation in cancer by using ultra-sensitive capillary electrophoresis-electrospray ionization-mass spectrometry (CE-ESI-MS) [30]. Global $5 \mathrm{hmC}$ levels of the tumor gDNA were markedly decreased compared to the adjacent healthy tissue gDNA, with an average of $85 \%$ and $64 \%$ reduction in colorectal 
A

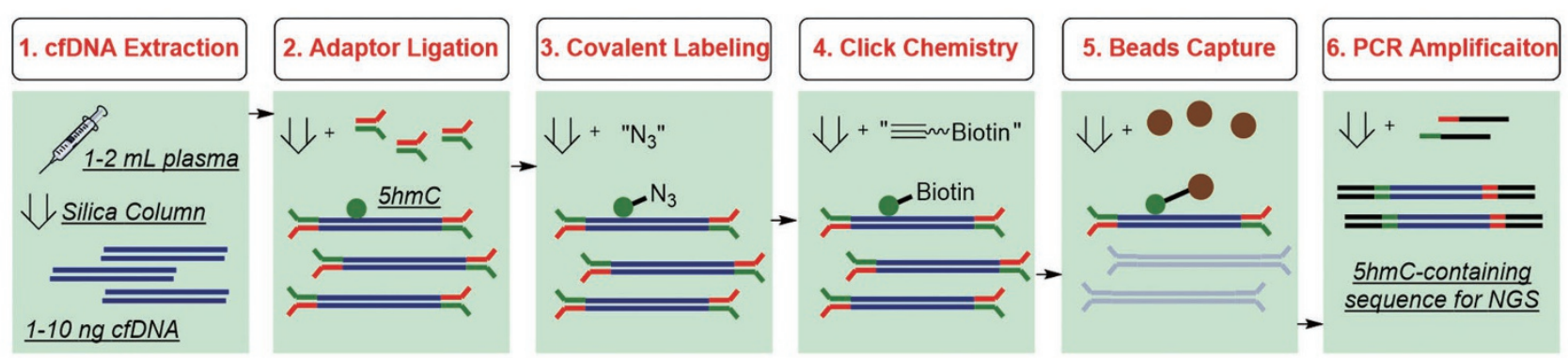

B
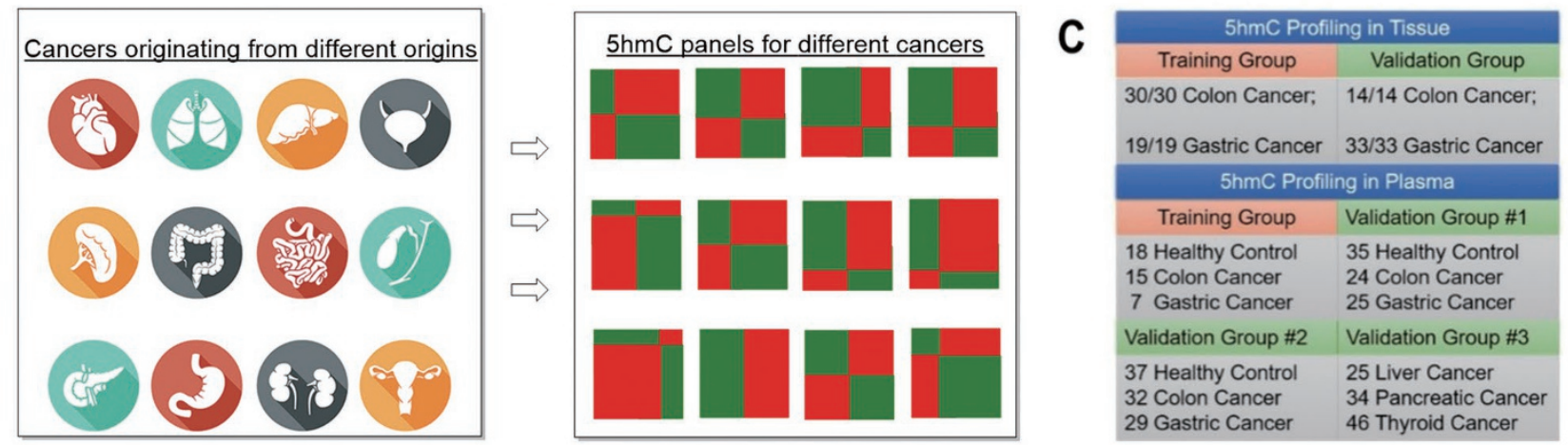

Figure 1 Detecting $5 \mathrm{hmC}$ biomarkers in cfDNA of human cancers. (A) Workflow of $5 \mathrm{hmC}$-Seal profiling from cfDNA is shown. Purified cfDNA is ligated with standard sequencing adaptors. $5 \mathrm{hmC}$-containing cfDNA fragments are selectively labeled with a biotin group. The biotin-labeled fragments are captured on the avidin beads, followed by PCR amplification and next-generation sequencing (NGS). (B) Cancers of different tissue origins (e.g., lung, colon, stomach, liver, ovary, pancreas) may release cfDNA decorated with distinct $5 \mathrm{hmC}$ modification patterns. Unique $5 \mathrm{hmC}$ signatures specific for different cancer types could be detected as biomarkers for diagnosis and prognosis. As shown in this panel, a unique 5hmC signature may correspond to each cancer type. (C) Schematic overview of sample collection, data generation and analysis is shown.

and gastric tumor samples, respectively. Global $5 \mathrm{hmC}$ levels of the cancer patients' plasma cfDNA showed a more limited decrease compared to control plasma cfDNA, consistent with low proportions of tumor-derived DNA in the total cfDNA pool (Supplementary information, Figure S2).

In plasma cfDNA, $5 \mathrm{hmC}$ was enriched within gene bodies and DNase I sensitive peaks whereas it was depleted at transcription start sites, $\mathrm{CpG}$ islands and transcription factor (TF) binding peaks relative to the flanking areas (Supplementary information, Figure S3A-S3F). This suggests an accumulation of $5 \mathrm{hmC}$ surrounding TFs at active transcription sites. $5 \mathrm{hmC}$ was also enriched in regions bearing permissive histone marks such as $\mathrm{H} 3 \mathrm{~K}$ 27ac, H3K4me1 and $\mathrm{H} 3 \mathrm{~K} 9 \mathrm{me} 1$, whereas it was underrepresented in regions bearing repressive markers such as H3K9me3 (Supplementary information, Figure S3G$\mathrm{S} 3 \mathrm{R}$ ). The genomic enrichment pattern of $5 \mathrm{hmC}$ was consistently observed in tissues gDNA (Supplementary information, Figure S3) and was similar between disease and healthy samples (Supplementary information, Figure S4).
Differential $5 \mathrm{hmC}$ loci associated with colorectal cancer

The average $5 \mathrm{hmC}$ profiles of plasma cfDNA were distinct from those of tissue and blood cell gDNA (Figure 2A), which could be due to their distinct cell origins and/ or the different DNA degradation properties in cell-free circulation. Among gDNA profiles, variations attributable to tissue identity (colon and stomach tissues, white blood cells) were dominant over variations attributable to disease status (healthy individual vs cancer patient, tumor vs adjacent tissue). Across plasma cfDNA profiles, colorectal and gastric cancer samples were more closely related with each other than with healthy controls (Figure 2A).

We compared $5 \mathrm{hmC}$ profiles from plasma cfDNA between 15 colon cancer patients and 18 healthy controls in the discovery batch to identify differentially modified $5 \mathrm{hmC}$ loci. The profiles were separated into 18 feature categories: gene bodies, promoters, $\mathrm{CpG}$ islands and cis-regulatory elements delineated by the Encyclopedia of DNA Elements (ENCODE) [31]. A parallel analysis compared $5 \mathrm{hmC}$ profiles from gDNA between colorectal tumors and adjacent tissues in 30 patients in the tissue discovery batch. All feature categories showed enrich- 
A

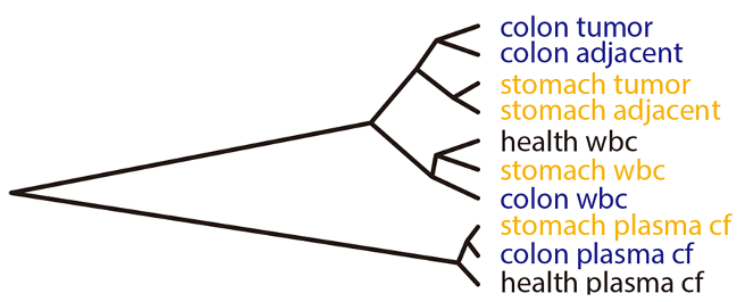

C

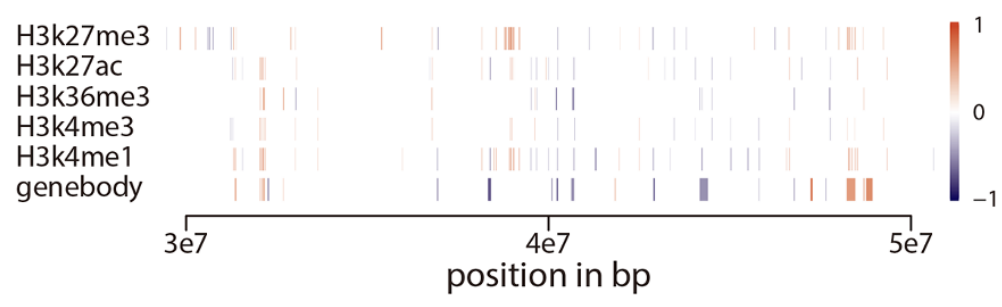

D

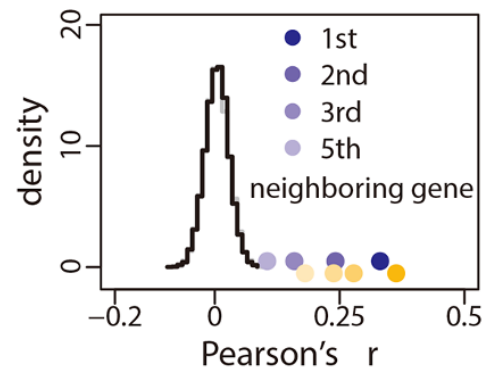

E $\quad$ - $5 \mathrm{hm} C$ density

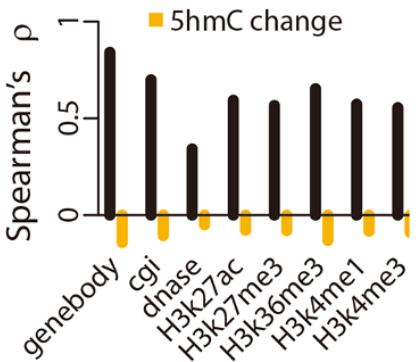

B
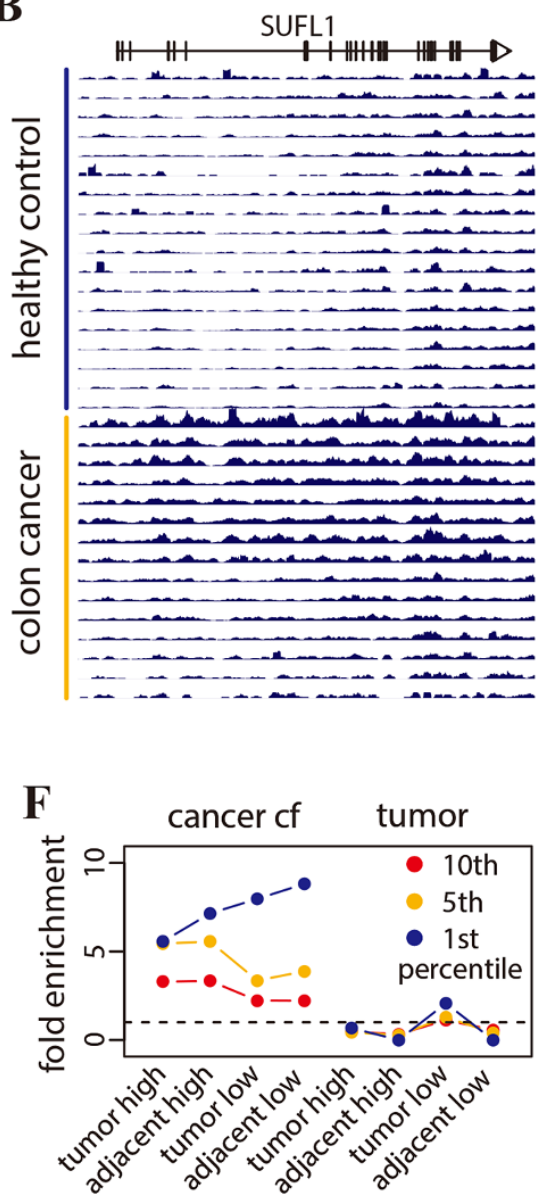

Figure 2 Differential $5 \mathrm{hmC}$ loci associated with cancer. (A) Average $5 \mathrm{hmC}$ levels in gene bodies in healthy controls (health) and cancer patients (colon, stomach), estimated for plasma cfDNA (plasma cf), white blood cell genomic DNA (WBC) and tissue genomic DNA (tumor, adjacent), were clustered by correlation distance. (B) Counts per million reads at SULF1 gene (plus $\pm 20 \mathrm{~kb}$ region) in plasma cfDNA of the 15 healthy controls and 18 colorectal cancer patients. The moving averages at 0.01 smoother span are shown. (C) The distribution of colorectal cancer-associated $5 \mathrm{hmC}$ loci detected at $5 \%$ false discovery rate in plasma cfDNA. Each vertical bar denotes a differential locus (a histone modification peak or a gene body). The color key indicates the relative magnitude of $\log _{2}$ fold change in cancer patients vs controls. (D) Pearson's correlation of $\log _{2}$ fold changes between all analyzed genes and their neighboring genes (points) was plotted against the null distribution of correlation with their first neighboring genes (curves), generated by shuffling gene positions for 1000 times. Blue and orange points denote data from plasma cfDNA and tissue gDNA, respectively, for colorectal cancer. In $\mathbf{C}$ and $\mathbf{D}$, chromosome 1 is shown as an example. (E) Cancer plasma cfDNA and tumor gDNA exhibit correlation in average 5hmC density (library size and feature length normalized $\log _{2}$ counts, black bars). However, there is no correlation in the $\log _{2}$ fold changes between differential $5 \mathrm{hmC}$ loci detected (between cancer vs health (plasma cfDNA)) and tumor vs adjacent tissue (tissue gDNA), (orange bars). (F) Genes with a $5 \mathrm{hmC}$ level elevated in cancer plasma cfDNA (cancer cf) are enriched in genes with high $5 \mathrm{hmC}$ level in tissue gDNA (tumor high, adjacent high). To estimate fold enrichment, the 1st, 5th and 10th percentile genes in descending order of the $\log _{2}$ fold change in cancer cfDNA were compared against the corresponding percentile genes in descending order of the average $5 \mathrm{hmC}$ level in tissue gDNA. Similarly, genes with a $5 \mathrm{hmC}$ level decreased in cancer plasma, and cfDNA are enriched in genes with low $5 \mathrm{hmC}$ levels in tissue gDNA (tumor low, adjacent low). In contrast, differentially marked genes detected in tumor gDNA (tumor) show no such enrichment pattern. Dashed line denotes no enrichment.

ment of differentially modified $5 \mathrm{hmC}$ loci (Supplementary information, Table S4). Figure 2B shows a differential locus detected in plasma cfDNA at the SULF1 (sulfatase 1) gene. In cancer patients' plasma cfDNA, the $5 \mathrm{hmC}$ levels in SULF1 were elevated in both exons and introns, with a peak pattern similar to that of tissue gDNA (Supplementary information, Figure S5). Neighboring differential $5 \mathrm{hmC}$ loci showed regionally elevated or 
decreased $5 \mathrm{hmC}$ levels (Figure $2 \mathrm{C}$ ). For example, the correlation of cancer-associated $5 \mathrm{hmC}$ changes between neighboring genes was significantly higher than a null distribution generated by shuffling gene positions within a chromosome (Figure 2D). This may suggest that $5 \mathrm{hmC}$ modifications occur and change in a relatively long-range, region-wise pattern.

The $5 \mathrm{hmC}$ densities across genomic features showed a correlation between cancer plasma cfDNA and tumor gDNA samples (Spearman's $\rho$ 0.34-0.84, Figure 2E). This locus-specific correlation of the $5 \mathrm{hmC}$ density is expected because of biological constraints. In contrast, we found no correlation between cancer patients' plasma cfDNA and tumor gDNA for cancer-associated $5 \mathrm{hmC}$ changes (Figure 2E). This can be explained by the greater variation in $5 \mathrm{hmC}$ levels among different tissues than that between disease status (Figure 2A). When gDNA from tumor tissue is released into plasma and mixed with the vast amount of background cfDNA derived from a variety of different tissues, the additional tumor signal observed at a given locus would be determined by the order of locus-, tissue- and disease-specific variations. Consistent with this expectation, we found that genes with $5 \mathrm{hmC}$ level elevated in cancer patients' plasma cfDNA were enriched in genes with high $5 \mathrm{hmC}$ levels in the tumor tissue gDNA. The top $1 \%$ of genes with the most elevated $5 \mathrm{hmC}$ levels in cancer patients' plasma cfDNA were enriched by over five-fold in the top $1 \%$ of genes with the greatest $5 \mathrm{hmC}$ levels in tumor and adjacent tissues (Fisher's exact tests $P<0.001)$. Similarly, genes with $5 \mathrm{hmC}$ levels decreased in cancer patients' plasma cfDNA were enriched in genes with low $5 \mathrm{hmC}$ levels in tumor and adjacent tissues (Figure $2 \mathrm{~F}$ ). In contrast, no such enrichment pattern was observed for the differentially modified $5 \mathrm{hmC}$ loci detected in tumor gDNA (Figure 2F).

\section{Classification of colorectal cancer by $5 \mathrm{hmC}$ markers de- rived from plasma cfDNA}

Unsupervised hierarchical clustering using differentially modified $5 \mathrm{hmC}$ loci derived from plasma cfDNA generally separated colorectal cancer patients from healthy individuals in the validation batch (Figure $3 \mathrm{~A}$ ). Across the various feature categories, the $\log _{2}$ fold change of $5 \mathrm{hmC}$ levels in gene bodies showed the greatest correlation between the discovery and validation batches (Spearman's $\rho=0.79$, Figure 3B), indicating that $5 \mathrm{hmC}$ loci in gene bodies are potentially more stable cancer biomarkers. We selected 989 differential loci in gene bodies detected at a $5 \%$ false discovery rate (FDR) with a greater than 1.2 -fold change (increase or decrease in cancer; Supplementary information, Table S5) for cancer classification. A model-based classifier that applies elastic net regularization on logistic regression was trained using the discovery samples (15 patients vs 18 controls) and then tested in the validation samples (24 patients vs 35 controls). Receiver operating characteristic (ROC) curves were generated to evaluate the performance using the area under the curve (AUC). The prediction algorithm achieved $83 \%$ sensitivity and $94 \%$ specificity (AUC $=0.95$, Figure $3 \mathrm{C}$ ) for patient classification.

An additional validation batch (32 patients vs 37 controls) was independently collected and tested, achieving $88 \%$ sensitivity and $89 \%$ specificity (AUC $=0.94$, Figure 3D). The classifier was further tested on a set of US samples (Supplementary information, Table S2), all of European descent, collected at the University of Chicago Medical Center. The classifier detected 4 out of 5 patients as cancer positive ( $80 \%$ sensitivity) and called 1 out of 6 healthy controls ( $83 \%$ specificity) in this small cohort. Therefore, although the classifier was trained on Chinese patients, it could capture a general signal of $5 \mathrm{hmC}$ changes in plasma cfDNA in colorectal cancer.

For comparison, we applied similar approaches to evaluate the performance of $5 \mathrm{hmC}$ biomarkers derived from colorectal tumor tissues. We selected 219 differential loci at gene bodies called at 5\% FDR and 1.2-fold change between tumor and adjacent tissues (Supplementary information, Table S6) from 30 patients of the discovery batch. The $5 \mathrm{hmC}$ tissue biomarkers showed a sensitivity of $86 \%$ and a specificity of $100 \%$ (AUC $=0.96$, Figure $3 \mathrm{E})$ in 14 patients from the tissue validation batch, suggesting that the $5 \mathrm{hmC}$ biomarkers from plasma cfDNA exhibit performance comparable to that from tissue gDNA. The colorectal cancer classifiers derived from plasma cfDNA and tissue gDNA profiles are detailed in Supplementary information, Table S7.

Disease sensitivity and specificity of plasma cfDNA-derived $5 \mathrm{hm} C$ markers

We next assessed the ability of the $5 \mathrm{hmC}$ biomarkers derived from plasma cfDNA to classify cancer stages in a subset of patients with available records. The $5 \mathrm{hmC}$ classifier assigned incremental numbers of cancer individuals (predicted cancer probability $>0.5$ ) for patients having undergone surgery for treatment $(0 / 2)$, patients at cancer stage I (4/6) and patients at stage II and III (38/40) (Cochran-Armitage test for trend $P=0.00038$, Figure $3 \mathrm{~F}$ ). The classifier had good but reduced power to call Stage IV patients (12/18), who in general suffered from metastasis to various tissues and are expected to have more complex tumor DNA profiles in circulation due to metastasis.

We further assessed disease specificity of the classifier in patients with colon-related benign diseases $(n=49)$ 
A

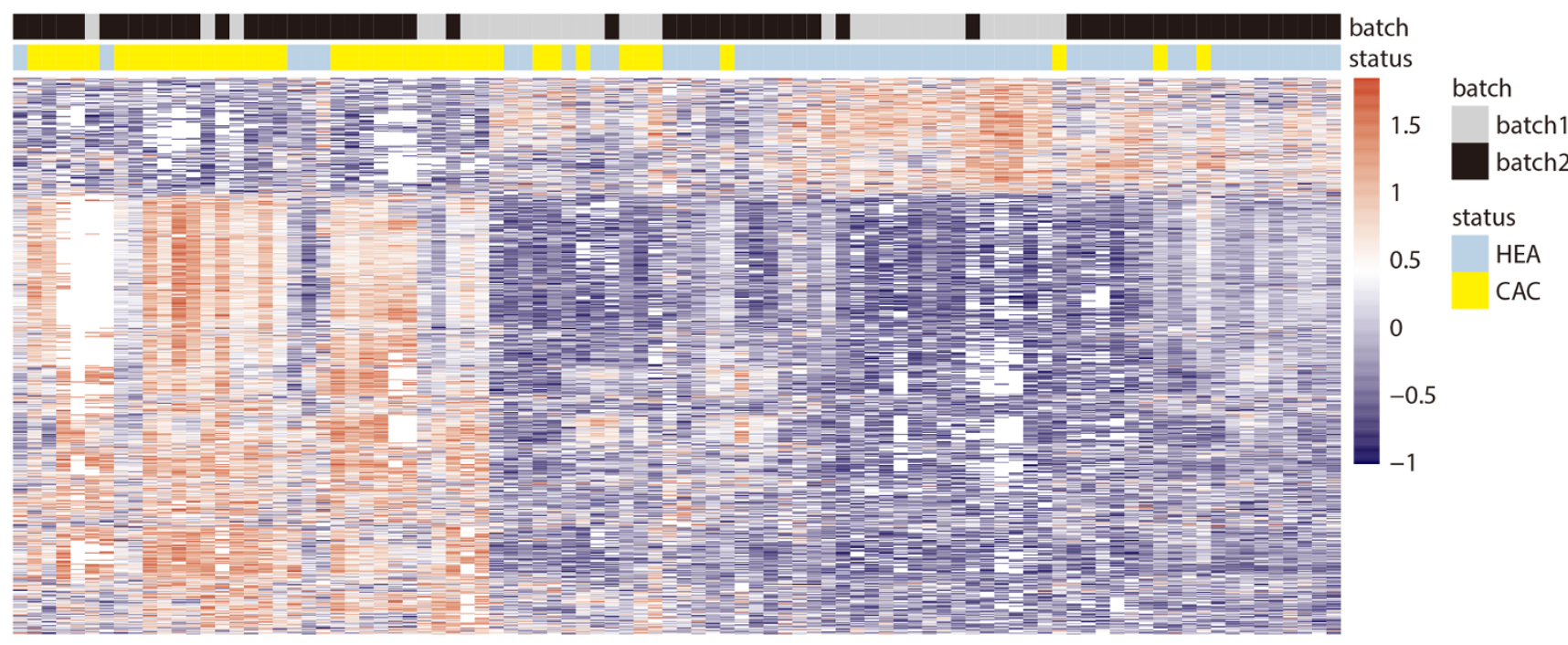

B

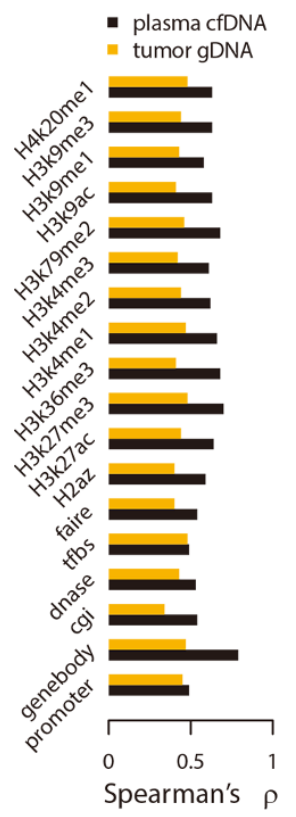

C

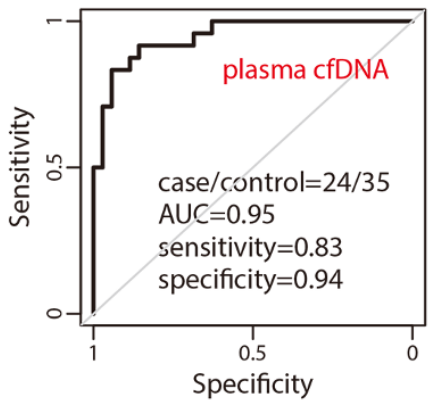

F

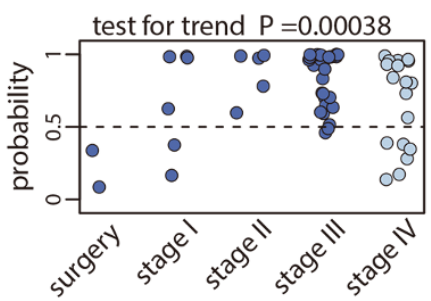

D

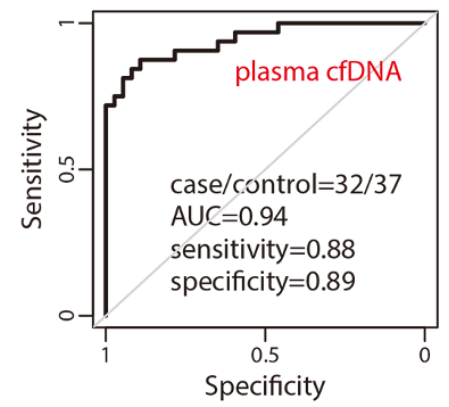

E

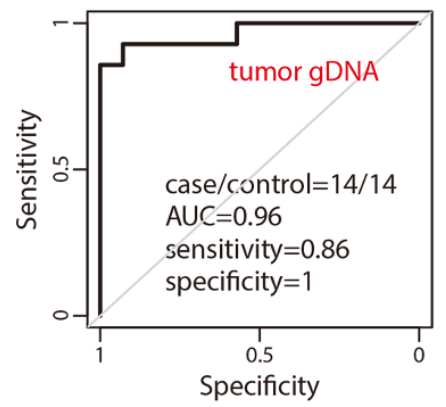

G

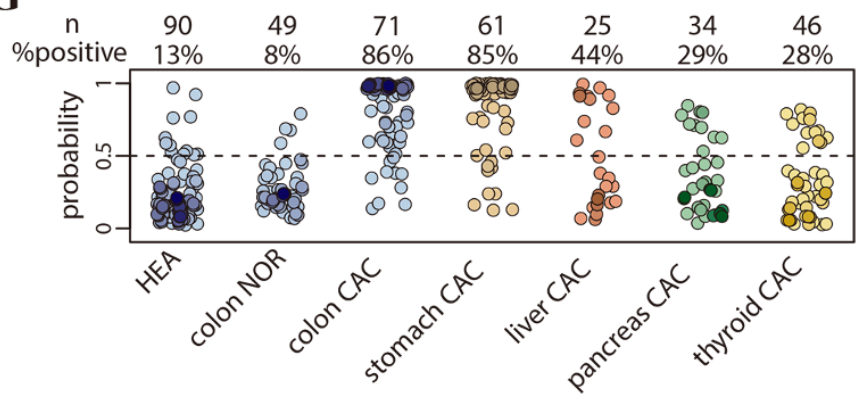

Figure 3 Performance of $5 \mathrm{hmC}$ biomarkers for colorectal cancer. (A) The heat map shows clustering of cfDNA samples from both the discovery and validation batches, using the 989 differential gene bodies detected in plasma cfDNA from the discovery batch. (B) Correlation of $5 \mathrm{hmC}$ changes in cancer between the discovery and validation batches is higher in plasma cfDNA (cancer patients vs healthy individuals) than in tissue gDNA (tumors vs adjacent tissues), especially for $5 \mathrm{hmC}$ in gene bodies. (C, D) Classification of two independent validation batches using $5 \mathrm{hmC}$ classifier derived from plasma cfDNA from the discovery batch. (E) Classification of an independent set of colon cancer tumor tissues using $5 \mathrm{hmC}$ biomarkers detected from the discovery batch of tissue samples (tumors vs adjacent tissues). (F) The predicted cancer probability (i.e., score) based on $5 \mathrm{hmC}$ classifier from plasma cfDNA shows a significant trend associated with clinical stage. Patients after surgery show predicted scores undistinguishable from healthy individuals. (G) The $5 \mathrm{hmC}$ cfDNA classifier for colorectal cancer is disease- and potentially cancer type-specific, showing decreasing predicted probability in cfDNA from stomach, liver, pancreatic and thyroid cancer patients. AUC, area under curve; CAC, cancer patients; HEA, healthy controls; NOR, patients with benign tumor. 
and patients with colorectal $(n=71)$, gastric $(n=61)$, liver $(n=25)$, pancreatic $(n=34)$ and thyroid $(n=46)$ cancer. Compared to the $86 \%$ call rate in colorectal cancer patients, only $8 \%$ of patients with benign colon diseases were predicted as having cancer (Figure 3G). The classifier also demonstrated certain tissue specificity, with a decreasing cancer call rate in gastric (85\%), liver (44\%), pancreatic (29\%) and thyroid (28\%) cancer patients (Figure $3 \mathrm{G})$. The lower sensitivity in calling the other cancers is not due to any intrinsic difficulty in classifying those cancers, as we achieved a much greater sensitivity in liver and pancreatic cancer using $5 \mathrm{hmC}$ markers derived from plasma cfDNA from patients with these respective cancers (data not shown). These results indicate that distantly related cancers can be readily distinguished by the corresponding classifiers through joint testing, while classification of closely related cancers such as colorectal and gastric cancer may be facilitated by additional diagnostic criteria.

A subset of cancer patients had records of classical biomarkers and epidemiological risk factors (Supplementary information, Table S1), with which we compared plasma cfDNA-derived $5 \mathrm{hmC}$ biomarkers for cancer detection sensitivity. The detection sensitivity of carcinoembryonic antigen (CEA, 32\%), alpha-fetoprotein (AFP, 0\%), carbohydrate antigen 125 (CA125, 13\%), CA15-3 (0\%), CA19-9 (19\%), CA72-4 (17\%), cytokeratin 19 (49\%), neuron-specific enolase (NSE, 21\%), and relationship to overweight (body mass index $\geq 25 \mathrm{~kg} / \mathrm{m}^{2}, 34 \%$ ), smoking (9\%), alcohol consumption (7\%) and previous history of cancer $(0 \%)$ were all less than $50 \%$. By calling cancer if any conventional biomarker or risk factor is positive, the upper bound detection sensitivity of the combined classical biomarkers and epidemiological factors only reached $54 \%$, a sensitivity rate much lower than the $86 \%$ that we could achieve using $5 \mathrm{hmC}$ markers. In addition, compared with the methylated SEPT9 (septin 9) gene, a blood-based epigenetic biomarker for colon cancer, our cfDNA $5 \mathrm{hmC}$ biomarkers registered a significantly further improved overall sensitivity ( 0.86 vs 0.48 based on public data) [32].

\section{$5 \mathrm{hmC}$ markers derived from plasma cfDNA in gastric cancer}

Next, we analyzed gastric cancer using plasma cfDNA samples. In the discovery batch, $5 \mathrm{hmC}$ loci in 7 gastric cancer patients were compared to 18 healthy controls across genomic feature categories (Supplementary information, Table S4). Using the detected differential $5 \mathrm{hmC}$ loci, 25 gastric cancer patients could be generally separated from 35 healthy individuals in the validation batch (Figure 4A). Again, $5 \mathrm{hmC}$ changes in gene bodies showed relatively higher correlations between the discovery and validation batches compared to other genomic features (Figure 4B). A model-based classifier was generated using the 1431 differential loci in gene bodies identified at 5\% FDR and 1.2-fold change in the discovery batch (Supplementary information, Table S8). This was applied to the validation batch, achieving 92\% sensitivity and $91 \%$ specificity (AUC $=0.93$, Figure $4 C$ ). Further assessment of the gastric cancer classifier in an additional, independently collected validation batch (29 patients vs 37 controls) achieved $90 \%$ sensitivity and $97 \%$ specificity (AUC $=0.97$, Figure 4D). The classification performance of the $5 \mathrm{hmC}$ biomarkers derived from cancer cfDNA was comparable to that from tumor gDNA samples: a classifier based on 161 differential $5 \mathrm{hmC}$ loci in gene bodies detected in 19 pairs of tumors and adjacent tissues in the discovery batch (Supplementary information, Table S9) was applied on 33 pairs of tissues in the validation batch, achieving $82 \%$ sensitivity and 94\% specificity (AUC = 0.93 , Figure 4E). The gastric cancer classifiers derived from plasma cfDNA and tissue gDNA profiles are detailed in Supplementary information, Table S10.

The $5 \mathrm{hmC}$ gastric cancer classifier derived from plasma cfDNA showed a trend of increasing cancer call rate with cancer clinical stage $(P=0.11$, Figure $4 \mathrm{~F})$. The classifier also demonstrated disease and tissue specificity, with $0 \%$ cancer call rate for benign gastric diseases, and with decreasing cancer call rate in patients with colorectal $(61 \%)$, liver $(28 \%)$, pancreatic $(6 \%)$ and thyroid $(0 \%)$ cancer patients (Figure 4G).

The detection sensitivity of classical biomarkers and epidemiological factors for gastric cancer was 13\% (CEA), 6\% (AFP), 6\% (CA125), 3\% (CA15-3), 14\% (CA19-9), 29\% (CA72-4), 36\% (cytokeratin 19), 13\% (NSE), 18\% (overweight), 25\% (smoking), 10\% (alcohol) and 7\% (previous history of cancer) (Supplementary information, Table S1). The upper bound sensitivity combining these markers and factors was $70 \%$, which again is lower than the $80 \%$ call rate observed in our studies.

\section{Tissue origin of the cancer-associated $5 \mathrm{hmC}$ changes ob-} served in plasma cfDNA

To demonstrate the tumor relevance of the plasma cfDNA from cancer patients, we sought to examine its source in patient-derived xenograft (PDX) mouse models. PDX mouse models were derived from tumors of three colorectal patients and three gastric patients, each with three independent xenograft animals. Plasma cfDNA of PDX mice was collected at 12-15 weeks of age, from which the $5 \mathrm{hmC}$-containing fragments were enriched and sequenced using the same protocol as with human plasma cfDNA. The proportion of cfDNA derived from the 


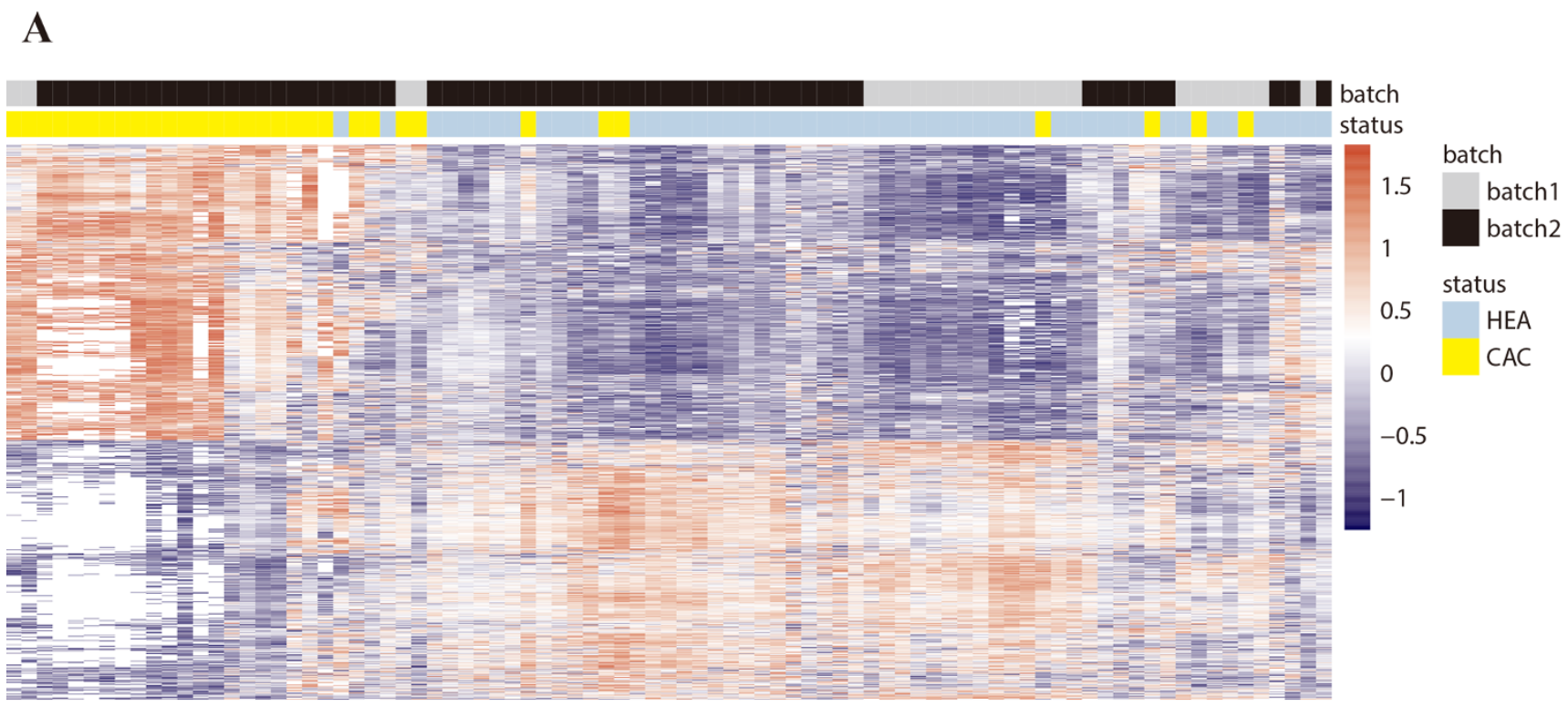

B

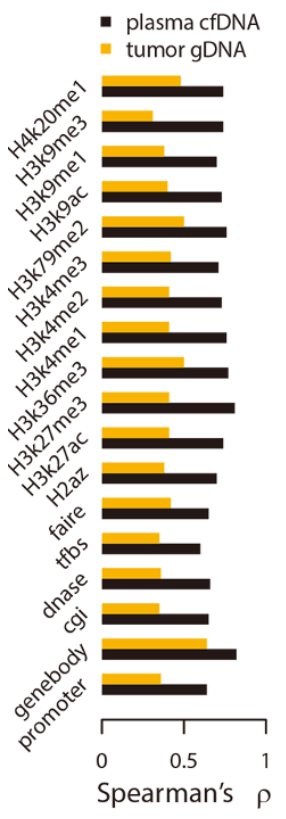

C

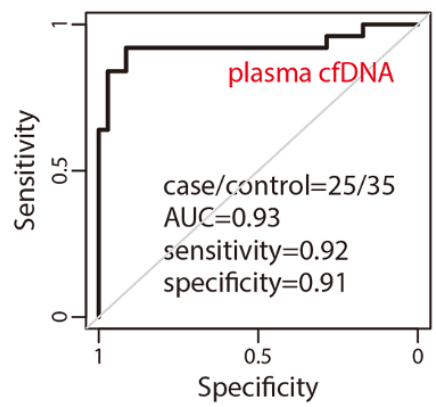

F

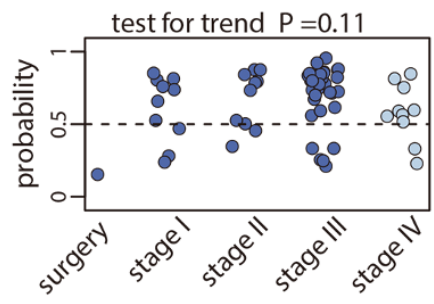

D

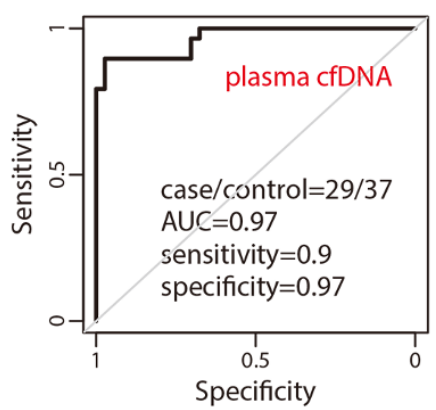

E

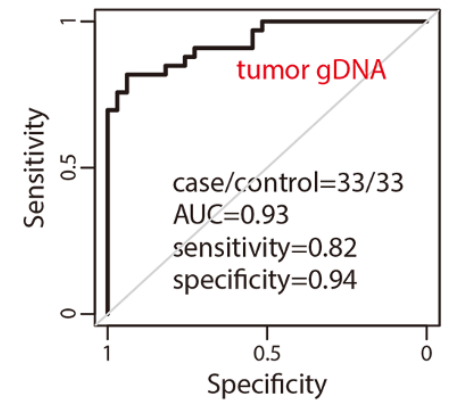

G

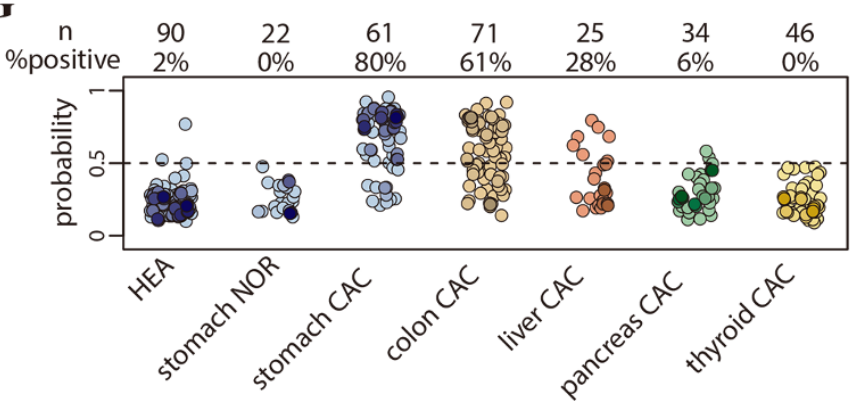

Figure 4 Performance of $5 \mathrm{hmC}$ biomarkers for gastric cancer. (A) The heat map shows clustering of cfDNA samples from both the discovery and validation batches, using the 1431 differential gene bodies detected in plasma cfDNA from the discovery batch. (B) Correlation of $5 \mathrm{hmC}$ changes in cancer between the discovery and validation batches is higher in plasma cfDNA (cancer patients vs healthy individuals) than in tumor gDNA (tumors vs adjacent tissues), especially for $5 \mathrm{hmC}$ in gene bodies. (C, D) Classifying two independent validation batches using $5 \mathrm{hmC}$ classifier derived from plasma cfDNA from the discovery batch. (E) Classifying an independent set of gastric cancer tumor tissues using 5 hmC biomarkers detected from the discovery batch of tissue samples (tumors vs adjacent tissues). (F) The predicted cancer probability (i.e., score) based on the $5 \mathrm{hmC}$ classifier from plasma cfDNA shows a trend associated with clinical stage. The one patient after surgery shows a predicted probability undistinguishable from healthy individuals. (G) The $5 \mathrm{hmC}$ cfDNA classifier for gastric cancer is diseaseand potentially cancer type-specific, showing decreasing predicted probability in cfDNA from colorectal, liver, pancreatic and thyroid cancer patients. 
tumor, estimated as the proportion of sequencing reads uniquely mapped to the human genome, was significantly increased in mice grafted with gastric tumors $(P=0.0020)$ and showed an increased trend in mice grafted with colorectal tumors with fewer passages ( $P=0.16$, Figure 5A).

Only the sequencing reads mapped to the human genome were further analyzed. Pearson's correlation of $5 \mathrm{hmC}$ profile between plasma cfDNA of PDX mice and gDNA of donor tumors significantly depends on the number of passages $(P=0.037$, Figure $5 \mathrm{~B})$. This suggests a quantitative relationship between tumor growth and the experimental capture of tumor $5 \mathrm{hmC}$ in plasma cfDNA, as the size $(P=0.0096)$ and growth rate $(P=0.0080)$ of tumors grafted in PDX mice increase with passage numbers (Figure 5B). Using the top five genes with the greatest $5 \mathrm{hmC}$ levels in PDX plasma cfDNA, donor tumor and the derived PDX from the same individual patient were clustered together (Figure 5C), indicating donor tumor tissue as the human origin of the PDX cfDNA.

PDX allowed us to study tumor-derived cfDNA without confounding background cfDNA. Genes with greater $5 \mathrm{hmC}$ levels in tumor-sourced PDX plasma cfDNA were
A

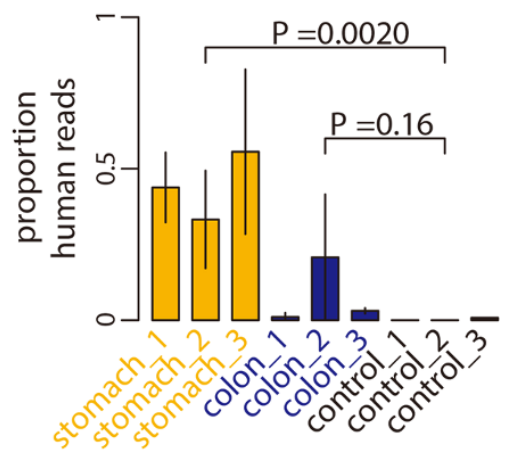

C

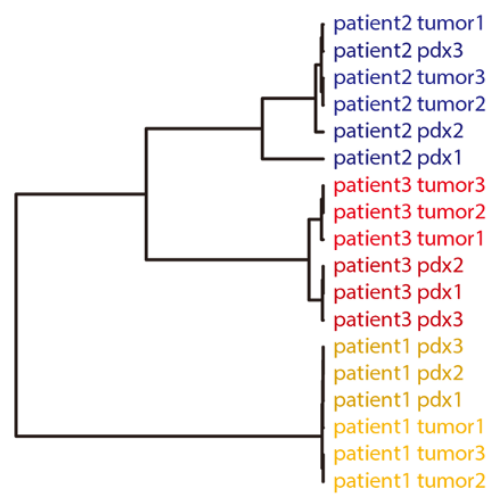

B

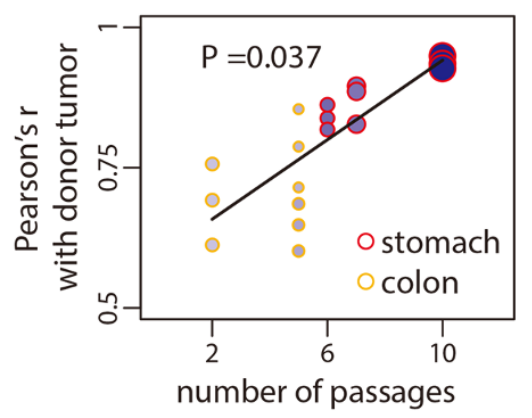

D

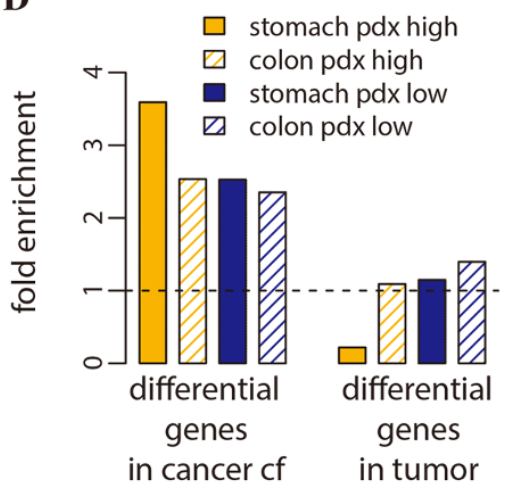

Figure 5 The origin of cancer-associated $5 \mathrm{hmC}$ changes observed in plasma cfDNA. (A) The proportions of human reads in plasma cfDNA captured with $5 \mathrm{hmC}$-Seal are shown for PDX mice grafted with tumor from three gastric cancer patients (stomach_1-3), three colorectal cancer patients (colon_1-3) and for PDX mice without graft (control_1-3). Vertical bars represent s.d. estimated from three replicate PDX mice for each patient. The PDX mice grafted with gastric tumor had greater number of passages (6-10) than those grafted with colorectal tumor (2-5). (B) The correlation of the $5 \mathrm{hmC}$ profile between tumor-derived, PDX plasma cfDNA and donor tumor gDNA depends on the number of passages of the PDX mouse. The size of the points is proportional to the size of grafted tumor, and the density of color denotes the growth rate of the grafted tumor. (C) Using the correlation distance of the top five genes that had the greatest $5 \mathrm{hmC}$ level in PDX plasma cfDNA, donor tumor gDNA and PDX plasma cfDNA from the same individual patient were clustered together. (D) Genes with elevated $5 \mathrm{hmC}$ level in cancer patient plasma cfDNA are enriched in genes with high $5 \mathrm{hmC}$ levels in PDX plasma cfDNA. To estimate fold enrichment, the fifth percentile genes in descending order of the $\log _{2}$ fold change in patient cfDNA were compared against the corresponding fifth percentile genes in descending order of the $5 \mathrm{hmC}$ level in PDX mice (stomach pdx high, colon pdx high). Similarly, genes with decreased $5 \mathrm{hmC}$ level in cancer patient plasma cfDNA are enriched in genes with low $5 \mathrm{hmC}$ levels in PDX plasma cfDNA (stomach pdx low, colon pdx low). Dashed line denotes no enrichment. 
more likely to be the genes with elevated $5 \mathrm{hmC}$ level in plasma cfDNA in cancer patients. Indeed, we found that genes with increased $5 \mathrm{hmC}$ levels in patient plasma cfDNA were enriched in those genes with greater $5 \mathrm{hmC} \mathrm{lev-}$ els in PDX plasma cfDNA, whereas genes with decreased $5 \mathrm{hmC}$ levels in patient plasma cfDNA were enriched in genes with lower $5 \mathrm{hmC}$ levels in PDX plasma cfDNA (Fisher's exact tests $P<1 \times 10^{-9}$, Figure 5D). In contrast, genes with $5 \mathrm{hmC}$ level changed between tumor and adjacent tissues showed no such enrichment pattern (Figure 5D).

\section{Tumor-associated $5 \mathrm{hmC}$ changes in gene regulation}

To investigate the potential functional role of $5 \mathrm{hmC}$ in gene regulation, we evaluated the relationship between gene expression changes and $5 \mathrm{hmC}$ level changes in tumors in two colorectal and one gastric cancer patients. We performed an RNA-seq assay in tumor tissues and paired adjacent tissues. The $\log _{2}$ fold changes of gene expression and the $\log _{2}$ fold change of $5 \mathrm{hmC}$ level in tumors relative to adjacent tissues were estimated across the three patients. Gene dysregulation and $5 \mathrm{hmC}$ changes were then compared across a combined list of 200 differential $5 \mathrm{hmC}$ loci in gene bodies detected in colorectal and gastric tumors in the discovery batches. The correlation between gene expression changes and $5 \mathrm{hmC}$ changes in tumors is highly significant $\left(P=9.8 \times 10^{6}\right.$, Supplementary information, Figure S6A). In addition, genes with altered $5 \mathrm{hmC}$ levels in cancer plasma cfDNA or in tumor gDNA were enriched in cancer- and metastasis-related pathways [33] (Supplementary information, Figure S6B).

\section{Discussion}

In general, $5 \mathrm{hmC}$ marks active loci because gene activation requires removal of the repressive $5 \mathrm{mC}$ methylations. It occurs in gene bodies of activated genes as well as at various enhancers, indicating that the genomic locations of $5 \mathrm{hmC}$ reflect gene activation at permissive chromatin (Supplementary information, Figure $\mathrm{S} 3$ ). $5 \mathrm{hmC}$ is chemically stable and thus its locations in gDNA can be stored in fragmented cfDNA for potential detection using non-invasive sampling. Utilizing a robust and highly efficient profiling-based approach to map $5 \mathrm{hmC}$ in plasma cfDNA samples from patients with cancer, we were able to identify $5 \mathrm{hmC}$ biomarkers that can distinguish cancer patients from healthy individuals with high sensitivity and specificity for colorectal (Figure 3 ) and gastric (Figure 4) cancers. Our study revealed the genome-wide pattern of cancer-associated $5 \mathrm{hmC}$ changes in plasma cfDNA (Figure 2) and demonstrated its tumor origin using PDX models (Figure 5). The identified $5 \mathrm{hmC}$ biomarkers can be cancer type-specific (Figures 3 and 4). The optimization of this approach with additional patient studies in future will further improve its performance and expand the scope of its application to other cancers. The strategy presented here provides a foundation for effective future biopsy-based diagnosis using body fluids and potentially prognostic indicators of human disease progression using cfDNA.

The dynamics of cancer cfDNA turnover is yet largely unknown. Under what is likely to be a simplified model, gDNA of tumor tissue is released into plasma and undergoes degradation reaching an equilibrium similar to that of background cfDNA from normal healthy tissues. Locus-specific $5 \mathrm{hmC}$ modification appears to be the primary determinant of $5 \mathrm{hmC}$ levels, with tissue specificity and then the cancer state adding additional layers of variation. These tissue-, and to a lesser extent, cancer-specific signals in DNA released from tumor tissues slightly shift the $5 \mathrm{hmC}$ modification profile of background plasma cfDNA toward that of tumor tissue gDNA. The more cfDNA released from tumor tissues, the greater of the shift giving increased power to discriminate the biological and clinical variation of the tumor source. Therefore, it will be critical to integrate a panel of $5 \mathrm{hmC}$ profiles from gDNA of diverse tissue types to achieve future assessment of disease specificity with cancer biomarkers. In addition, solid tumors are composed of carcinoma stem cells and carcinoma cells, within a microenvironment constituted by leucocytes, cells of mesenchymal origin and extracellular matrix [34]. Tumor progression initiates a gradient of change in the local environment characterized by hypoxia and vascularization. Extensive variability may exist within a growing tumor and its surrounding cells such that certain types of cells are prone to apoptosis and to releasing DNA to the circulation. We expect that cancer-associated changes in $5 \mathrm{hmC}$ observed in plasma cfDNA were contributed by distinct sets of cells within or surrounding the tumor tissues. Single-cell or cell-type-specific $5 \mathrm{hmC}$ profiling of tumor-associated tissues and using appropriate cell type markers, would reveal the extent and distribution of the cell specificity of these modificaitons and shed further light on the properties of the source cells that contribute to the cancer-associated $5 \mathrm{hmC}$ changes observed in plasma cfDNA. It is our intent to pursue these future directions.

\section{Materials and Methods}

\section{Study design}

Patient population A total of 180 patients older than 18 years with colorectal (CC), gastric (GC) cancers and hepatocellular carcinoma were diagnosed at three different medical centers in 
Shanghai Huashan Hospital at Fudan University, China from September 2015 to July 2016. Eighty patients with thyroid cancer and pancreatic cancer were diagnosed at Peking Union Medical College Hospital, China during 2014-2016. The population was socioeconomically diverse, most patients coming from Beijing and East China (the City of Shanghai and the Provinces of Zhejiang, Jiangsu, and Anhui). All specimens were collected from patients who were newly diagnosed or having postoperative recurrence, as well as from two postoperative colorectal cancer patients and one postoperative gastric cancer patient, and had the tumor status confirmed by histological evaluation. Patients treated with chemotherapy, radiation therapy or immunotherapy were excluded from this study. In total, this study was conducted among 80 colorectal cancer patients and 75 gastric cancer patients, with an additional 25 hepatocellular carcinoma patients, 34 pancreatic cancer patients and 46 thyroid cancer patients to explore cancer type specificity. Whole blood samples from 90 healthy individuals under physical examination were also collected at Fudan University, China during September 2015-May 2016 as healthy controls; these individuals are Han Chinese and showed no history of cancer and had no abnormalities in laboratory examinations. However, the follow-up data for all patients were unavailable because of the short follow-up time. Informed consent was obtained from each participating subject before the study, which was approved by the Institutional Review Board (IRB) at each collaborating institution.

Batch design To minimize the influence of any batch effect, gastrointestinal participants were assigned into three batches in chronological order of enrollment in the study. Differential $5 \mathrm{hmC}$ features between cancer patients and controls were analyzed with batch 1 (discovery set) and validated in batches 2 and 3 (validation sets).

Sample overview Detailed information about the study subjects is shown in Supplementary information, Tables S1 and S2, including number of samples, gender, age, clinical diagnosis, stage classified according to the tumor, node and metastasis (TNM) guidelines (version 7), and eight conventional cancer biomarkers that were measured in patients. Four common tumor markers for gastrointestinal cancer screening are CEA, AFP, CA19-9 and CA72-4, which were positive in $0 \%, 33.8 \%, 21.4 \%$ and $16.9 \%$ of CC, as well as $7.5 \%, 13.6 \%, 14.3 \%$ and $29.0 \%$ of GC patients, respectively.

Additional US samples for validation Five patients with colorectal cancer were diagnosed at the University of Chicago Medical Center (UCMC) from September 2015 to July 2016. All samples were collected from patients who were newly diagnosed and had no distal metastasis at the time that blood was taken. Whole blood samples from six healthy individuals, who are non-Hispanic or Latino individuals of European ancestry, under physical examination were also collected at the UCMC during September 2015May 2016 as healthy controls. Informed consent was obtained from each participating subject before the study, which was approved by the IRB at the University of Chicago.

\section{Preparation of cfDNA samples}

cfDNA samples were prepared from peripheral blood collected from patients and healthy controls. Briefly, $4 \mathrm{ml}$ of peripheral blood was collected from each subject using EDTA anticoagulant tubes, and the plasma sample was prepared within $6 \mathrm{~h}$ by centrifuging twice at $1350 \times \mathrm{g}$ for $12 \mathrm{~min}$, and then centrifuging at 13 $500 \times g$ for $12 \mathrm{~min}$. The prepared plasma samples (about $2 \mathrm{ml} / \mathrm{sub}-$ ject) were immediately stored at $-80{ }^{\circ} \mathrm{C}$. The plasma cfDNA was isolated using the QIAamp Circulating Nucleic Acid Kit (Qiagen) according to the manufacturer's protocol. Within each experimental batch, samples were randomized with respect to disease status in the following library preparation and sequencing profiling.

\section{Isolation of gDNA from tissues}

Tissue samples, including tumor and adjacent tissue samples, from patients were stored at $-80{ }^{\circ} \mathrm{C}$ after surgical removal. About 10-25 mg tissue was collected using a scalpel after sample thawing. gDNA from tissues was isolated using the ZR Genomic DNA Tissue Kits (Zymo Research) according to the manufacturer's protocol.

\section{5hmC-Seal-seq library preparation and sequencing}

Seal-seq libraries for $5 \mathrm{hmC}$ profiling were prepared following our previously patented technology [29]

In this method, the T4 bacteriophage $\beta$-glucosyltransferase is used to transfer an engineered glucose moiety containing an azide group onto the hydroxyl group of $5 \mathrm{hmC}$ across the human genome. The azide group is then chemically modified with biotin for affinity enrichment of $5 \mathrm{hmC}$-containing DNA fragments. First, the gDNA is fragmented using an enzymatic reaction. Next, the fragmented gDNA or the cfDNA were repaired and installed with the Illumina compatible adaptors. The glucosylation reactions were performed in a $25 \mu \mathrm{l}$ reaction containing $50 \mathrm{mM}$ HEPES buffer (pH 8.0), $25 \mathrm{mM} \mathrm{MgCl}_{2}$, purified DNA, $100 \mu \mathrm{M} \mathrm{N}_{3}$-UDP-Glc and $1 \mu \mathrm{M} \beta \mathrm{GT}$ at $37^{\circ} \mathrm{C}$ for $1 \mathrm{~h}$. The reaction mix was subject to purification using a Micro Bio-Spin 30 Column (Bio-Rad) into $\mathrm{dd}_{2} \mathrm{O}$. Subsequently, $1 \mu 1$ DBCO-PEG4-DBCO (Click Chemistry Tools, $4.5 \mathrm{mM}$ stock in DMSO) was added to the reaction mixture. The reactions were incubated at $37^{\circ} \mathrm{C}$ for $2 \mathrm{~h}$. Next, the DNA was purified ona Micro Bio-Spin 30 Column (Bio-Rad). The purified DNA was incubated with $5 \mu \mathrm{l}$ C1 Streptavidin beads (Life Technologies) in $2 \times$ buffer $(1 \times$ buffer: $5 \mathrm{mM}$ Tris pH 7.5, $0.5 \mathrm{mM}$ EDTA, $1 \mathrm{M}$ $\mathrm{NaCl}$ ) for 15 min according to the manufacturer's instruction. The beads were subsequently washed eight times for 5 min with $1 \times$ buffer. All binding and washing was done at room temperature with gentle rotation. The captured DNA fragments were amplified with 14-16 cycles of PCR amplification. The PCR products were purified using AMPure XP beads according to the manufacturer's instructions. The DNA concentration of each library was measured with a Qubit fluorometer (Life Technologies) and sequencing was performed on the Illumina Hi-Seq or NextSeq 500 platform.

\section{RNA-seq library preparation and sequencing}

Tumor and adjacent tissue samples including two colon samples and one stomach sample were collected and RNA was isolated using the ZR-Duet DNA/RNA Miniprep Kit (Zymo Research). Total isolated RNA was used to construct the library with the NEBNext Ultra RNA Library Prep Kit for Illumina following the manufacture's protocol. Sequencing reactions were executed on the NextSeq 500 platform using paired-end mode, yielding at least 32 $\mathrm{M}$ reads per sample. 
Establishment of patient-derived tumor xenografts

The animal protocol for this study was reviewed and approved by the Ethical Committee of Medical Research, Shanghai Huashan Hospital of Fudan University. It utilized 6-8 week old BALB/c nu/ nu mice weighing 16-20 g on receipt (Shanghai Laboratory Animal Center). The fresh pathological tissue fragments were placed in sterile tissue culture medium on ice and brought immediately to the animal facility. Tumor graft samples were cut into multiple $1 \times 1 \times$ $1 \mathrm{~mm}$ fragments in complete media. Tumor material was implanted into female BALB/c nu/nu mice under isoflurane anesthesia; all possible efforts were made to minimize suffering. A skin incision $(0.3 \mathrm{~cm})$ was subsequently made on the right mid-back. One tumor piece $(1-3 \mathrm{~mm})$ was inserted into each pocket and the skin was closed. Mice were regularly checked. When thetumor diameter reached $1.5 \mathrm{~cm}$, mice were euthanized and tumors were excised, cut into $1 \times 1 \times 1 \mathrm{~mm}$ fragments again, and passaged to successive generations of three mice. The remaining tumor was snap frozen in liquid nitrogen and stored at $-80{ }^{\circ} \mathrm{C}$; plasma was also separated from blood sampled via the mouse eyeball. In this study, the gastric cancer and colorectal cancer patient-derived tumor xenografts (PDX) were randomly selected in our existing PDX model library, while the control group was BALB/c nu/nu mice of 12-14 weeks old.

\section{5 hm C enrichment analysis}

We designed two similar spike-in probes with unique sequences, named $5 \mathrm{hmC}$ spike-in and no5hmC spike-in.

5hmC spike-in:

5'-CTGTCATGGTGACAAAGGCATCC* GGCAGAAATGCCCACACAGCCTCTTTAACCAGCACGCCAACCGCCTCTGCTTCGGCCCTGGTCACGCAGCTGACAAGGTCTTCATAATAGAGAAATCCTG-3', C* -5 hmC modifications.

no5hmC spike-in:

5'-CTGTCATGGTGACAAAGGCATCGCAGCGAAATGCCCACACAGCCTCTTTAACCAGCACGCCAACCGCCTCTGCTTCGGCCCTGGTCACGCAGCTGACAAGGTCTTCATAATAGAGAAATCCTG-3'

These sequences cannot map to the human reference genome. Six cfDNA sequencing libraries were constructed from the same cfDNA (10 ng) sample, which were divided into control and experimental groups, each having three duplicates. About 100 million copies of $5 \mathrm{hmC}$ and no5hmC spike-ins were then mixed with the experiment sample before library preparation. The control group did not include the $5 \mathrm{hmC}$ pull-down step, whereas the experimental group included the $5 \mathrm{hmC}$ pull-down procedure. After sequencing, we extracted spike-in reads and calculated the enrichment ratios. The average ratio of $5 \mathrm{hmC}$ spike-in to no5hmC spike-in in the control group was 0.72 , while the ratio in the experimental group was 40.36 .

\section{Technical stability analysis for $5 \mathrm{hm} C$ Seal-seq library preparation}

The designed spike-in probes were utilized to improve the robustness and sensitivity of $5 \mathrm{hmC}$-Seal. In total 20000 copies of $5 \mathrm{hmC}$ and no5hmC spike-ins were pre-mixed and then added into the same cfDNA samples before constructing libraries. Different spike-in samples were designed as follows: $20 \mathrm{ng}$ cfDNA with 2 repeats, 10 ng cfDNA with 10 repeats, 5 ng cfDNA with 2 repeats, 2 ng cfDNA with 2 repeats, 1 ng cfDNA with 2 repeats.
Total $5 \mathrm{hm} C$ quantification in cfDNA and $g D N A$

The enzymatic digestion protocol for each gDNA and cfDNA sample was the same. gDNA or cfDNA (all in $8 \mu \mathrm{H}_{2} \mathrm{O}$ ) was first denatured by heating at $95{ }^{\circ} \mathrm{C}$ for $5 \mathrm{~min}$ and then transferred into ice water for $2 \mathrm{~min}$. Next, $1 \mathrm{~L}$ of $10 \times \mathrm{S} 1$ nuclease buffer $(30 \mathrm{nM}$ $\left.\mathrm{CH}_{3} \mathrm{COONa}, \mathrm{pH} 4.6,260 \mathrm{mM} \mathrm{NaCl}, 1 \mathrm{mM} \mathrm{ZnSO}_{4}\right)$ and 180 units (1 $\mu 1)$ of $\mathrm{S} 1$ nuclease were added into the DNA solution. The mixture $(10 \mu \mathrm{l})$ was then incubated at $37^{\circ} \mathrm{C}$ for $4 \mathrm{~h}$. Then $34.5 \mu \mathrm{l}$ of $\mathrm{H}_{2} \mathrm{O}$, $5 \mu \mathrm{l}$ of $10 \times$ alkaline phosphatase buffer $(50 \mathrm{mM}$ Tris- $\mathrm{HCl}, 10 \mathrm{mM}$ $\left.\mathrm{MgCl}_{2}, \mathrm{pH} 9.0\right), 0.5 \mu \mathrm{l}$ of alkaline phosphatase were added into the DNA digestion solution. The incubation was continued at $37{ }^{\circ} \mathrm{C}$ for an additional $4 \mathrm{~h}$.

The CE-ESI-MS experiments were carried out with the CESI8000 capillary electrophoresis (CE) system from Beckman Coulter coupled with a Sciex Tripel Quad 5500 Mass Spectrometer (Sciex) through a modified Nanosprayed II interface. Bare fused silica capillaries etched with porous tips were made available by Beckman Coulter; these could be inserted into the sheathless nanospray interface. The separation capillary was $100 \mathrm{~cm}$ long with an internal diameter of $30 \mu \mathrm{m}$ and an outside diameter of $150 \mu \mathrm{m}$. The capillary was flushed with methanol for $10 \mathrm{~min}$ at $100 \mathrm{psi}$, followed by water, $0.1 \mathrm{M}$ sodium hydroxide, $0.1 \mathrm{M}$ hydrochloric acid and water for $10 \mathrm{~min}$ each at $100 \mathrm{psi}$, and finally by the background electrolyte (BGE) of $10 \%$ acetic acid $(\mathrm{pH} \mathrm{2.2)} \mathrm{for} 10 \mathrm{~min}$ at 100 psi before first use. The BGE was also used as conductive liquid in the conductive liquid capillary. Before each run, the conductive liquid capillary was rinsed with BGE for 5 min at 100 psi. Samples for detection were stored at $5{ }^{\circ} \mathrm{C}$ in the $\mathrm{CE}$ system. Hydrodynamic injections were used in this study, and about $100 \mathrm{nl}$ sample was injected into the separation system for each analysis. A voltage of $+25 \mathrm{kV}$ was applied during the separation and the current was between 3.0 and $3.2 \mu \mathrm{A}$. The electrospray voltage was optimized to get the best nanospray stability and efficiency, and $+1.7 \mathrm{kV}$ was good enough for this study. The quantification calibration curves of $5^{\prime}-\mathrm{dC}, 5^{\prime}-\mathrm{mdC}$ and $5^{\prime}$-hmdC were constructed using mixture solution of their standards in different concentration. The resulting solutions of our DNA samples were directly measured by CE-ESIMS. The concentrations of these three nucleosides in each sample were calculated based on the calibration curves. And the $5^{\prime}-\mathrm{mdC} /$ $\mathrm{dC}$ and $5^{\prime}-\mathrm{hmdC} /\left(\mathrm{dC}+5^{\prime}-\mathrm{mdC}\right)$ results of each sample were then calculated.

\section{Sequencing data processing and detection of differential loci}

Read-through sequences within raw sequencing reads were trimmed using Trimmomatic version 0.35 [35]. Low-quality bases at the $5^{\prime}$ (Phred quality score $<5$ ) and 3' (5 bp-sliding window Phred score $<15$ ) were also trimmed. Reads with a minimum length of $50 \mathrm{bp}$ were aligned to the human genome assembly GRCh37 using Bowtie2 version 2.2.6 [36] with the end-to-end alignment mode. For paired-end sequencing data, read pairs were concordantly aligned with fragment length $<500 \mathrm{bp}$ and with up to 1 ambiguous base, and four mismatched bases per $100 \mathrm{bp}$ length. Alignments with Mapping Quality Score (MAPQ) $\geq 10$ were counted for overlap with genomic features using featureCounts of subread version 1.5.0-p1 [37], without strand information. Autosomal feature counts with $>10$ mean counts across samples were then normalized and compared between-group using DESeq2 version 1.12.3 [38]. Since gender is not a significant covariate for both autosomal gene expression [39] and DNA methylation [40], while 
aging has been linked to DNA methylation [41], age at sample collection/surgery was included as a categorical variable $(<20,20-55$, $>55 \mathrm{yr}$ ) in the negative binomial generalized linear model implemented in DESeq2. As for the experimental batches (discovery and validation sets), samples were processed in less than 1 week by 1-3 technicians, the identity of the technician was included in the model to adjust for potential technical correlation. When comparing tumor and adjacent tissues, patient identity was nested under technician identity. A FDR [42] of 5\% was used to identify differential $5 \mathrm{hmC}$ loci.

For PDX mouse plasma cfDNA data, sequencing reads were trimmed and aligned to a composite assembly of mixed human and mouse $(\mathrm{GRCm} 38)$ genome. Unique alignments (MAPQ $\geq 10)$ were separated into human and mouse reads by chromosome name.

For RNA-seq data, sequencing reads were trimmed and aligned to GRCh37 annotated with GENCODE release 19, using STAR version 2.5.1b [43]. Unique alignments with $\geq 90 \%$ match over reads were summarized by featureCounts. For the correlation analysis presented in Supplementary information, Figure S6A, 5hmC data were also summarized over exon regions as in RNA data. For genes having $>10$ mean counts across samples, $\log _{2}$ fold change between tumor and adjacent tissues were estimated by DESeq2 adjusting for patient identity.

\section{Refining $5 \mathrm{hmC}$ biomarkers and evaluating performance}

Cancer prediction models were trained using the differential $5 \mathrm{hmC}$ loci (e.g., gene bodies) detected in the discovery batch. We applied elastic net regularization on a logistic linear regression model [44], using the glmnet library in the R Statistical Package [44]:

$$
\log \frac{\operatorname{Pr}(G=1 \mid x)}{\operatorname{Pr}(G=0 \mid x)}=\beta_{0}+x^{T} \beta
$$

Where $x$ is a $J \in(1 \ldots j)$ by $I \in(1 \ldots i)$ matrix of $5 \mathrm{hmC}$ level at gene $j$ for sample $i$. The model is solved by

$$
\begin{aligned}
& \min _{\left(\beta_{0}, \beta\right)}\left[\frac{2}{2 N} \sum_{i=1}^{N}\left(y_{i}-\beta_{0}-x_{i}^{T} \beta\right)^{2}+\lambda P_{\alpha}(\beta)\right] \\
& P_{\alpha}(\beta)=\sum_{j=1}^{p} v_{j}\left[\frac{1}{2}(1-\alpha) \beta_{j}^{2}+\alpha\left|\beta_{j}\right|\right],
\end{aligned}
$$

where $P_{\alpha}$ is the blend of the ridge $(\alpha=0)$ and the lasso $(\alpha=1)$ penalty. The parameter $\lambda$ controls for the overall strength of penalty, while the parameter $\alpha$ controls for the relative proportion between the ridge and lasso penalty. For a given $\alpha, \lambda$ is estimated by cross validation and selected as the largest $\lambda$ at which the mean cross-validated error is within one s.e. of the minimum. As we expect that $5 \mathrm{hmC}$ loci with a larger effect size are more robustly detected by the assay and therefore more reproducible across experiments, we included in the model a penalty factor $v_{j}$ so that

$$
v_{j}=\frac{1}{\left|f_{j}\right|},
$$

where $f_{j}$ is the $\log _{2}$ fold change of gene $j$ estimated in the training batch.

The parameter $\alpha$ reflects the model assumption (i.e., a large number of small effects or a small number of large effects). In small data sets like our discovery batch, the selection of $\alpha$ based on residual errors may lead to an over-fitted model. Instead, our model assumption was guided by the validation batch, so that $\alpha$ was searched to maximize AUC in the validation batch over a grid of values from 0.05 to 0.95 . The model, derived from the training batch using the selected $\alpha$, was applied in all classifications as described in Figures 3 and 4.

The normalization of data in cancer classification adopts the regularized log transformation implemented in DESeq2, which estimates a global mean dispersion trend to shrink the variance at low count genes that are associated with high Poisson noise, so that variance is stabilized across genes in the log transformed data. In Figure 3 and Supplementary information, Figure S6, data from the validation batches were all normalized to the reference distribution derived from the training batch and used directly in cancer classification, i.e., we essentially ignored any remaining batch effect, which could result from library preparation and the sequencing run. This is because under a real clinical setting, the batch effect estimated between testing samples and training samples will generally be biased due to the highly unbalanced case/control proportion in testing samples (low incidence of cancers). Batch effect may introduce some deviation from the 0.5 probability cutoff in cancer calling. External spike-ins may be used to estimate batch effect in future investigations.

ROC curves [45] were generated to evaluate the performance of a prediction algorithm, using the $p R O C$ [46] library in the $\mathrm{R}$ package. Sensitivity and specificity were estimated at the score cutoff that maximizes the sum of sensitivity and specificity using the $R O C R$ [45] library in the $\mathrm{R}$ package.

\section{Statistical analyses}

For Figure 5A, the $P$-value was estimated by a linear mixed effects model: proportion of human reads (square root transformed) $\sim$ xenograft status (none $\mid$ colorectal|gastric) $+\gamma$ (tumor donor identity) $+\varepsilon$, random effect $\gamma$ was introduced to control for correlation among replicate xenografts. For Figure 5B, $P$-value was estimated by a linear mixed effects model: Pearson's $r$ with tumor donor $~$ number of passages $+\gamma$ (tumor donor identity) $+\varepsilon$.

\section{Annotating $5 \mathrm{hmC}$ loci with genomic features and functional analysis}

The genomic features analyzed included promoters $(3 \mathrm{~kb}$ upstream of gene start), gene bodies (gene start to stop sites annotated by GENCODE release 24) [47], CpG islands (annotated CpG islands by UCSC Table Browser plus $+/ 1 \mathrm{~kb}$ region), and the ENCODE [31] features (DNase I hypersensitive sites, TF binding sites and histone modifications) [31]. Each type of ENCODE feature from the profiled ENCODE cell lines was integrated into a single list of features by collapsing overlapped and nearby $(<150$ bp) peaks. The ENCODE features analyzed in the $5 \mathrm{hmC}$ genomic distribution (Supplementary information, Figures S2 and S3) were as originally annotated without collapsing, with 20000 features randomly sampled in the interquartile size distribution for each feature category. Pathway enrichment analysis of genes with cancer patient-associated $5 \mathrm{hmC}$ loci were explored based on the Kyoto Encyclopedia of Genes and Genomes (KEGG) [48] using the NIH/ DAVID tool [49].

\section{Data availability}

All of the raw and processed data used in this study have been uploaded to the NCBI Sequence Read Archive (SRP080977) and 
Gene Expression Omnibus (GSE89570) depositories. The R code related to classifier detection and modeling is available upon request.

\section{Acknowledgments}

This work was supported in part, by grants from the National Institutes of Health (U01CA217078 to MBB and WZ, R33CA225338 to $\mathrm{CH}$ and WZ, P30CA060553 Career Development Fund to WZ, R01HG006827 to CH), The University of Chicago Ludwig Center for Metastasis Research (to $\mathrm{CH}$ ), and the Research Special Fund for Public Welfare Industry of Health (201402001 to YZ). We thank Dr H Pickersgill (Life Science Editors) for editing this manuscript.

\section{Author Contributions}

Conceptualization: YZ, WZ, CH and JL; Methodology: XL, YS, JN, FY, LW, GJ; Investigation: XL, YS; Formal analysis: XZ and WZ; Resources: ZL, JZ, WZ, DX, YW, YD, SY, JH, JS, HH, FL, LH, PW, XQ, MC, TZ, QL, MD, ZL, GC, KM, SA, MB; Writing original draft: WL, XZ, XL, LY, WZ and CH; Writing, review and editing, $\mathrm{YZ}$ and JL; Funding acquisition: $\mathrm{YZ}, \mathrm{WZ}$ and $\mathrm{CH}$.

\section{Competing Financial Interests}

The University of Chicago has filed for patent protection on the original $5 \mathrm{hmC}$-Seal technology in 2012. CH was one of the inventors. XL and YS are the shareholders of a company that has licensed the technology for clinical applications. The remaining authors declare that they have no competing interests.

\section{References}

1 Jaenisch R, Bird A. Epigenetic regulation of gene expression: how the genome integrates intrinsic and environmental signals. Nat Genet 2003; 33 Suppl:245-254.

2 Branco MR, Ficz G, Reik W. Uncovering the role of 5-hydroxymethylcytosine in the epigenome. Nat Rev Genet 2011; 13:7-13.

3 Taby R, Issa JP. Cancer epigenetics. CA Cancer J Clin 2010; 60:376-392.

4 Hussain S, Aleksic J, Blanco S, Dietmann S, Frye M. Characterizing 5-methylcytosine in the mammalian epitranscriptome. Genome Biol 2013; 14:215.

5 Kriaucionis S, Heintz N. The nuclear DNA base 5-hydroxymethylcytosine is present in Purkinje neurons and the brain. Science 2009; 324:929-930.

6 Tahiliani M, Koh KP, Shen YH, et al. Conversion of 5-methylcytosine to 5-hydroxymethylcytosine in mammalian DNA by MLL partner TET1. Science 2009; 324:930-935.

7 Ito S, Shen L, Dai Q, et al. Tet proteins can convert 5-methylcytosine to 5-formylcytosine and 5-carboxylcytosine. Science 2011; 333:1300-1303.

8 He YF, Li BZ, Li Z, et al. Tet-mediated formation of 5-carboxylcytosine and its excision by TDG in mammalian DNA. Science 2011; 333:1303-1307.

9 Pfaffeneder T, Hackner B, Truss M, et al. The discovery of 5-formylcytosine in embryonic stem cell DNA. Angew Chem Int Ed 2011; 50:7008-7012.

10 Bachman M, Uribe-Lewis S, Yang X, Williams M, Murrell A,
Balasubramanian S. 5-Hydroxymethylcytosine is a predominantly stable DNA modification. Nat Chem 2014; 6:1049-1055.

11 Ito S, D'Alessio AC, Taranova OV, Hong K, Sowers LC, Zhang Y. Role of Tet proteins in $5 \mathrm{mC}$ to $5 \mathrm{hmC}$ conversion, ES-cell self-renewal and inner cell mass specification. Nature 2010; 466:1129-1133.

12 Ko M, Huang Y, Jankowska AM, et al. Impaired hydroxylation of 5-methylcytosine in myeloid cancers with mutant TET2. Nature 2010; 468:839-843.

13 Ficz G, Branco MR, Seisenberger S, et al. Dynamic regulation of 5-hydroxymethylcytosine in mouse ES cells and during differentiation. Nature 2011; 473:398-U589.

14 Guo JU, Su YJ, Zhong C, Ming GL, Song HJ. Hydroxylation of 5-methylcytosine by TET1 promotes active DNA demethylation in the adult brain. Cell 2011; 145:423-434.

15 Koh KP, Yabuuchi A, Rao S, et al. Tet1 and Tet2 regulate 5-hydroxymethylcytosine production and cell lineage specification in mouse embryonic stem cells. Cell Stem Cell 2011; 8:200-213.

16 Mooijman D, Dey SS, Boisset JC, Crosetto N, van Oudenaarden A. Single-cell $5 \mathrm{hmC}$ sequencing reveals chromosome-wide cell-to-cell variability and enables lineage reconstruction. Nat Biotechnol 2016; 34:852-856.

17 Pastor WA, Pape UJ, Huang Y, et al. Genome-wide mapping of 5-hydroxymethylcytosine in embryonic stem cells. Nature 2011; 473:394-397.

18 Song CX, Szulwach KE, Fu Y, et al. Selective chemical labeling reveals the genome-wide distribution of 5-hydroxymethylcytosine. Nat Biotechnol 2011; 29:68-72.

$19 \mathrm{Wu} \mathrm{H}, \mathrm{D}$ 'Alessio AC, Ito S, et al. Dual functions of Tet1 in transcriptional regulation in mouse embryonic stem cells. $\mathrm{Na}$ ture 2011; 473:U389-U578.

$20 \mathrm{Yu}$ M, Hon GC, Szulwach KE, et al. Base-resolution analysis of 5-hydroxymethylcytosine in the mammalian genome. Cell 2012; 149:1368-1380.

21 Booth MJ, Branco MR, Ficz G, et al. Quantitative sequencing of 5-methylcytosine and 5-hydroxymethylcytosine at single-base resolution. Science 2012; 336:934-937.

22 Han D, Lu X, Shih AH, et al. A highly sensitive and robust method for genomewide $5 \mathrm{hmC}$ profiling of rare cell populations. Mol Cell 2016; 63:711-719.

23 Konstandin N, Bultmann S, Szwagierczak A, et al. Genomic 5-hydroxymethylcytosine levels correlate with TET2 mutations and a distinct global gene expression pattern in secondary acute myeloid leukemia. Leukemia 2011; 25:1649-1652.

24 Lo YM, Tein MS, Lau TK, et al. Quantitative analysis of fetal DNA in maternal plasma and serum: implications for noninvasive prenatal diagnosis. Am J Hum Genet 1998; 62:768-775.

25 Alix-Panabieres C, Pantel K. Clinical applications of circulating tumor cells and circulating tumor DNA as liquid biopsy. Cancer Discov 2016; 6:479-491.

26 Chan KC, Jiang P, Chan CW, et al. Noninvasive detection of cancer-associated genome-wide hypomethylation and copy number aberrations by plasma DNA bisulfite sequencing. Proc Natl Acad Sci USA 2013; 110:18761-18768.

27 Wen L, Li J, Guo H, et al. Genome-scale detection of hypermethylated $\mathrm{CpG}$ islands in circulating cell-free DNA of hepatocellular carcinoma patients. Cell Res 2015; 25:1250-1264.

28 Guo S, Diep D, Plongthongkum N, Fung HL, Zhang K, 
Zhang K. Identification of methylation haplotype blocks aids in deconvolution of heterogeneous tissue samples and tumor tissue-of-origin mapping from plasma DNA. Nat Genet 2017; 49:635-642.

29 Song CX, Szulwach KE, Fu Y, et al. Selective chemical labeling reveals the genome-wide distribution of 5-hydroxymethylcytosine. Nat Biotechnol 2011; 29:68-72.

30 Yuan F, Zhang XH, Nie J, Chen HX, Zhou YL, Zhang XX. Ultrasensitive determination of 5-methylcytosine and 5-hydroxymethylcytosine in genomic DNA by sheathless interfaced capillary electrophoresis-mass spectrometry. Chem Commun 2016; 52:2698-2700.

31 ENCODE. An integrated encyclopedia of DNA elements in the human genome. Nature 2012; 489:57-74.

32 Church TR, Wandell M, Lofton-Day C, et al. Prospective evaluation of methylated SEPT9 in plasma for detection of asymptomatic colorectal cancer. Gut 2014; 63:317-325.

33 Padua D, Massague J. Roles of TGFbeta in metastasis. Cell Res 2009; 19:89-102.

34 Pattabiraman DR, Weinberg RA. Tackling the cancer stem cells - what challenges do they pose? Nat Rev Drug Discov 2014; 13:497-512.

35 Bolger AM, Lohse M, Usadel B. Trimmomatic: a flexible trimmer for Illumina sequence data. Bioinformatics 2014; 30:2114-2120.

36 Langmead B, Salzberg SL. Fast gapped-read alignment with Bowtie 2. Nat Methods 2012; 9:357-359.

37 Liao Y, Smyth GK, Shi W. featureCounts: an efficient general purpose program for assigning sequence reads to genomic features. Bioinformatics 2014; 30:923-930.

38 Love MI, Huber W, Anders S. Moderated estimation of fold change and dispersion for RNA-seq data with DESeq2. Genome Biol 2014; 15:550.

39 Zhang W, Bleibel WK, Roe CA, Cox NJ, Eileen Dolan M. Gender-specific differences in expression in human lymphoblastoid cell lines. Pharmacogenet Genomics 2007; 17:447450.

40 Moen EL, Zhang X, Mu W, et al. Genome-wide variation of cytosine modifications between European and African populations and the implications for complex traits. Genetics 2013; 194:987-996.
41 Jones MJ, Goodman SJ, Kobor MS. DNA methylation and healthy human aging. Aging Cell 2015; 14:924-932.

42 Benjamini Y, Yekutieli D. The control of the false discovery rate in multiple testing under dependency. Ann Stat 2001; 29:1165-1188.

43 Dobin A, Davis CA, Schlesinger F, et al. STAR: ultrafast universal RNA-seq aligner. Bioinformatics 2013; 29:15-21.

44 Friedman J, Hastie T, Tibshirani R. Regularization paths for generalized linear models via coordinate descent. J Stat Softw 2010; 33:1-22.

45 Sing T, Sander O, Beerenwinkel N, Lengauer T. ROCR: visualizing classifier performance in R. Bioinformatics 2005; 21:3940-3941.

46 Robin X, Turck N, Hainard A, et al. pROC: an open-source package for $\mathrm{R}$ and $\mathrm{S}+$ to analyze and compare ROC curves. BMC Bioinformatics 2011; 12:77.

47 Harrow J, Frankish A, Gonzalez JM, et al. GENCODE: the reference human genome annotation for The ENCODE Project. Genome Res 2012; 22:1760-1774.

48 Kanehisa M, Sato Y, Kawashima M, Furumichi M, Tanabe M. KEGG as a reference resource for gene and protein annotation. Nucleic Acids Res 2016; 44:D457-462.

49 Huang da W, Sherman BT, Lempicki RA. Bioinformatics enrichment tools: paths toward the comprehensive functional analysis of large gene lists. Nucleic Acids Res 2009; 37:1-13.

(Supplementary information is linked to the online version of the paper on the Cell Research website.)

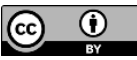

This work is licensed under a Creative Commons Attribution 4.0 Unported License. The images or other third party material in this article are included in the article's Creative Commons license, unless indicated otherwise in the credit line; if the material is not included under the Creative Commons license, users will need to obtain permission from the license holder to reproduce the material. To view a copy of this license, visit http:// creativecommons.org/licenses/by/4.0/

(C) The Author(s) 2017 